\title{
Score sonográfico predictor de éxito en el destete ventilatorio en la Unidad de Cuidados Intensivos
}

\author{
Alma Erika Hernández Plata, ${ }^{\star}$ Ma. Natalia Gómez González, ${ }^{*}$ Raúl Soriano Orozco, * \\ Jorge Adalid Díaz Rodríguez, ${ }^{*}$ Pedro Luis González Carrillo*
}

\section{RESUMEN}

Introducción: La ventilación mecánica (VM) ha sido fundamental en el manejo del paciente crítico. Cerca de $25 \%$ de los pacientes ventilados presentarán dificultades en el destete ventilatorio, su retraso está asociado a complicaciones diversas. En la actualidad se reconocen factores importantes del fracaso del destete ventilatorio, tal como la disfunción diafragmática que tras el surgimiento de la sonografía crítica se ha utilizado de manera más frecuente, encontrándose asociada a un incremento en los días de VM con un impacto en la mortalidad; a pesar de ello usualmente es infradiagnosticada. Se han establecido parámetros sonográficos predictores de éxito o fracaso en el destete ventilatorio; sin embargo, se encuentran como parámetros aislados, por lo que es necesaria la elaboración de modelos predictivos sonográficos para un destete ventilatorio exitoso.

Métodos: Se analizó en una primera etapa un total de 90 pacientes con ingreso a la unidad de cuidados intensivos bajo VM y protocolo de destete del mismo. Se realizaron mediciones clínicas, ventilatorias y sonográficas, siendo las de mayor peso estadístico las sonográficas; razón por la que se diseñó un score predictivo sonográfico para destete ventilatorio (SPSDV) consistente en cuatro rubros con puntuación máxima de 8 puntos y mínima de 0 . Se estableció el punto de mayor sensibilidad y especificidad para la prueba.

Resultados: Tras la aplicación y análisis estadístico del SPSDV se obtuvo una sensibilidad de $91 \%$ y especificidad de $69 \%$, con valor predictivo positivo y negativo de 89 y $75 \%$ respectivamente, con un área bajo la curva de 0.80 .

Conclusiones: El score predictivo sonográfico para destete ventilatorio es útil para identificar a pacientes con posible éxito de retiro.

Palabras clave: Destete ventilatorio mecánico, score predictivo sonográfico.

\section{SUMMARY}

Introduction: Mechanical ventilation has been fundamental in the management of critical patients. Almost $25 \%$ of patients ventilated will present difficulties in weaning process, which may be due to multiple causes. Delay or prolongation is associated with various complications. There are currently important factors for the failure of ventilatory weaning, such as diaphragmatic dysfunction, which has been consistently characterized following the advent of critical ultrasonography, it is associated with increase in days of ventilation and impact on mortality, despite of this facti, is underdiagnosed. Predictors of success or failure in weaning process has been established by means of diaphragmatic ultrasound; however, they are found as isolated parameters, so it is necessary to develop ultrasonic predictive models, in addition to the clinical ones, for a safe ventilatory weaning. Methods: A total of 90 patients with admission to the intensive care unit with mechanical ventilation and weaning protocol were analyzed in a first stage. Clinical, ventilatory and ultrasonographic measurements were performed, being the most statistically significant, ultrasonographic, and therefore an ultrasonographic predictive model was designed consisting of 4 items with a maximum score of 8 points and a minimum of 0 . It was established a point of greater sensitivity and specificity for the test.

Results: After the application and statistical analysis of the ultrasonographic predictive model, sensitivity of 0.91 and specificity of 0.69 were obtained, with positive and negative predictive value of $89 \%$ and $75 \%$, respectively, with ROC area of 0.80 .

Conclusions: The ultrasonographic predictive model, designed to identify patients with possible success, is also a predictor of the failure of mechanical ventilation.

Key words: Weaning mechanical ventilation, ultrasonographic model of sucess prediction.

\section{RESUMO}

Introdução: $A$ ventilação mecânica (VM) tem sido fundamental no manejo de pacientes em estado crítico. Quase $25 \%$ dos pacientes ventilados terão

\footnotetext{
* Unidad Médica de Alta Especialidad, Centro Médico Nacional Bajío Núm. 1. IMSS, León, Guanajuato.
}

Recepción: 01/09/2017. Aceptación: 05/09/2018.

Este artículo puede ser consultado en versión completa en http://www.medigraphic.com/medicinacritica dificuldades no desmame ventilatório, seu atraso está associado a várias complicações. Atualmente, fatores importantes são reconhecidos pelo fracasso do desmame ventilatório, como a disfunção diafragmática que após o surgimento da ultrassonografia crítica tem sido utilizado com maior frequência, estando associada a um aumento nos dias de VM, com impacto na mortalidade; apesar disso geralmente é subdiagnosticada. Estabelecemos parâmetros ultrassonográficos preditores de sucesso ou fracasso de desmame ventilatório, porém são parâmetros isolados sendo necessário o desenvolvimento de modelos ultrassonográficos preditivos para o sucesso do desmame ventilatório. Métodos: Em uma primeira etapa foram analisados um total de 90 pacientes internados na unidade de terapia com VM e protocolo de desmame. Medidas clínicas, ventilatórias e ultrassonográficas foram realizadas, sendo a de maior peso estatístico a ultrassonografia; portanto, foi desenhado um escore ultrassonográfico preditivo para o desmame ventilatório (SPSDV), composto por 4 itens com escore máximo de 8 pontos e escore mínimo de 0 . 0 ponto de maior sensibilidade e especificidade para o teste foi estabelecido.

Resultados: Após a aplicação e análise estatística do SPSDV, obteve-se sensibilidade de $91 \%$ e especificidade de $69 \%$ com valor preditivo positivo e negativo de $89 \%$ e $75 \%$, respectivamente, com área sob a curva de 0.80 . Conclusões: $O$ escore preditivo ultrassonográfico para o desmame ventilatório é útil para identificar pacientes com possível sucesso na retirada.

Palavras-chave: Desmame ventilatório mecânico, escore preditivo ultrassonográfico.

\section{INTRODUCCIÓN}

La ventilación mecánica (VM) sigue siendo una herramienta fundamental en el tratamiento de los pacientes en estado crítico, su objetivo es mantener una adecuada ventilación alveolar y un intercambio gaseoso efectivo, lo que ha permitido una mejoría en su pronóstico. ${ }^{1}$ Cerca de $25 \%$ de los pacientes bajo VM presentarán dificultades en el destete ventilatorio, ${ }^{2}$ lo cual puede deberse a múltiples causas. El retraso se vincula a complicaciones ampliamente conocidas como la neumonía asociada a la ventilación mecánica (NAVM), polineuropatía del paciente crítico entre otras, que derivan en un mal pronóstico y aumento de mortalidad hasta de $12 \%$, en comparación con los pacientes que no tienen dicho retraso, ${ }^{3}$ por tal motivo es importante el retiro del soporte ventilatorio lo antes posible. Más de la tercera parte del tiempo bajo VM se invierte tratando de destetar al paciente de dicho soporte. ${ }^{4}$ En la actualidad se reconocen factores importantes del fracaso del destete ventilatorio, a pesar de tener resueltas las causas que llevaron al uso de ventilación mecánica. Su presencia se asocia a tasas de bajo éxito en el progreso ventilatorio, tal es el caso de la disfunción diafragmática inducida por la ventilación (DDIV). ${ }^{5}$ La DDIV se define como un descenso progresivo de la fuerza muscular diafragmática que ocurre de forma precoz tras el inicio de la VM. ${ }^{6}$

La existencia de DDIV se asocia a un incremento en los días de VM hasta de 7-12 días con un impacto se- 
cundario en costos hospitalarios, pronóstico y mortali$\mathrm{dad}^{7}$ estimado en un estudio realizado por Young Kim et al., en una prevalencia de hasta $29 \%$ en la población de UCI. En este sentido, la función diafragmática en pacientes críticamente enfermos puede ser fácilmente comprometida, pues la mayoría está sometida a ventilación mecánica. Por lo anterior, la función diafragmática preservada es muy importante durante el proceso de destete para un adecuado inicio de respiración espontánea y su evaluación efectiva es una herramienta valiosa para la decisión del clínico.

La dificultad de estudiar dicha disfunción diafragmática se relaciona en parte con la complejidad de algunas técnicas de las que se dispone como la velocidad de conducción nerviosa frénica y fluoroscopia, ya que implican radiación ionizante y traslado que pueden poner en riesgo la estabilidad del paciente, ${ }^{8}$ además representan muchas limitaciones en el ámbito de la terapia intensiva y en la mayoría de los casos incluso no está disponible para la mayor parte de los centros hospitalarios. Por otra parte, se cuenta con el ultrasonido, el cual se ha consolidado como una herramienta sumamente valiosa en la terapia intensiva que puede realizarse a la cabecera del paciente. Además de accesible y no invasivo tiene gran eficacia diagnóstica para la evaluación de la cinética diafragmática y del esfuerzo inspiratorio.

La evaluación diafragmática ha sido objeto de investigaciones para predecir el éxito en el destete ventilatorio; tal es el caso del estudio de DiNino y cols. que determinó la sensibilidad y especificidad del grosor diafragmático (GD) y el porcentaje de variabilidad diafragmática (\%VD), tomando como punto de corte un porcentaje de variabilidad diafragmática de $\geq 30 \%$ en el éxito de la extubación, con una sensibilidad de $88 \%$ y especificidad de $71 \%$ y en grosor diafragmático se estableció que > $2.0 \mathrm{~mm}$ es predictor de éxito y por debajo de este valor puede asociarse a disfunción diafragmática, con una sensibilidad de $84 \%$ y especificidad de $18 \% .^{9}$

Se han descrito otros parámetros sensibles para correlacionar grado de disfunción diafragmática y éxito para el destete, tal es el caso de la excursión diafragmática (ED) con un valor $\geq 10 \mathrm{~mm}$, la cual fue estudiada por Kim y cols. observando en este parámetro una sensibilidad de $83 \%$ y especificidad de $41 \%$. Otro parámetro útil valorado en la ultrasonografía es la velocidad de contracción diafragmática (VCD) o fracción de acortamiento, la cual es dependiente de la excursión diafragmática y del tiempo inspiratorio.

A pesar del uso cada vez mayor de la sonografía en pacientes críticos, la disfunción diafragmática continúa siendo una patología infradiagnosticada. ${ }^{10}$ Estos parámetros sonográficos descritos ofrecen valoración integral de la función diafragmática en tiempo real, lo que facilita el seguimiento, ayudando a predecir el éxito de la técnica de retiro o indicando al clínico detener la ma- niobra y continuar con la VM. Sin embargo, estas cifras aisladas de cada parámetro pueden poner en duda el avance ventilatorio, por lo que es necesario establecer modelos predictivos para un destete ventilatorio exitoso que nos ofrezca alta sensibilidad y especificidad por medio de un puntaje de riesgo de fracaso o de éxito. Hasta el momento, no se ha desarrollado un modelo predictivo ultrasonográfico que conjunte todos los parámetros establecidos como "de buen pronóstico» para la extubación.

La razón del presente trabajo es realizar un score predictivo que conjunte todos estos parámetros establecidos y crear una herramienta para predecir el resultado del proceso de destete ventilatorio.

\section{MATERIALES Y MÉTODOS}

El presente estudio se realizó en dos etapas, la primera consistió en la revisión de la base de datos de la terapia intensiva del Centro Médico Nacional del Bajío en un periodo del 01 de noviembre de 2016 al 31 de marzo de 2017, se analizaron los expedientes clínicos electrónicos de 90 pacientes con ingreso a la UCI bajo ventilación mecánica y posterior protocolo de destete de la misma, en quienes se hubiera realizado sonografía diafragmática previo al retiro del VM.

Se analizaron variables clínicas, respiratorias y sonográficas, siendo estas últimas las mediciones más significativas. En estas mediciones sonográficas se incluyeron: a) grosor diafragmático, b) excursión diafragmática, c) velocidad de contracción diafragmática y d) porcentaje de variabilidad diafragmática. Los valores de predicción clínica y sonográfica para destete exitoso se analizaron mediante regresión logística multivariada y tablas de contingencia, obteniendo valores significativos en excursión diafragmática $(p=0.016)$; en porcentaje de variación diafragmática $(p=0.029)$ así como grosor diafragmático $(\mathrm{p}=0.001)$ y velocidad de contracción diafragmática $(p=0.01)$.

Con base en estos resultados se diseñó un modelo predictivo conjuntando dichos parámetros sonográficos, se les asignaron valores de referencia según lo obtenido en la primera etapa del estudio, los cuales correlacionaron con lo reportado en la literatura. ${ }^{1,2,4,9}$

En la segunda etapa se aplicó dicho score predictivo a los pacientes ingresados en la $\mathrm{UCl}$ en un periodo del 01 de abril de 2017 al 31 de julio de 2017, determinando la utilidad del score. Se asignó un valor total de 8 puntos máximos y mínimo de 0 para dicho score, tomando como punto de corte de 0 a 4 puntos como correlación de alto índice de fracaso y un puntaje mayor de 5 asociado a éxito.

El score sonográfico predictivo para destete ventilatorio aplicado a los pacientes durante este periodo se muestra en la Tabla 1. 
Los pacientes incluidos en este estudio cumplieron con los siguientes criterios: pacientes en $\mathrm{UCl}$, bajo ventilación mecánica, con diferentes diagnósticos médicos, que se encontraran en protocolo de destete ventilatorio y que cumplieran con los siguientes criterios clínicos, gasométricos y ventilatorios: $\mathrm{PaO}_{2} \geq 60 \mathrm{mmHg}$, $\mathrm{PaCO}_{2}: \leq 50 \mathrm{mmHg}, \mathrm{FiO}_{2}: \leq 50 \%$, relación $\mathrm{PaO}_{2} / \mathrm{FiO}_{2}$ : $\geq 200 \mathrm{mmHg}$. PEEP $\leq 5 \mathrm{cmH}_{2} \mathrm{O}, \mathrm{FR}: \leq 30$ respiraciones por minuto. Volumen tidal $\geq 5 \mathrm{~mL} / \mathrm{kg}$, vol. corriente $\geq 10 \mathrm{~mL} / \mathrm{kg}$. Mayores de 18 años de edad.

Los pacientes excluidos para el presente estudio fueron quienes, dentro de los diagnósticos quirúrgicos, se les hubiera realizado cirugía gástrica, esofágica o torácica (debido a la manipulación diafragmática intraoperatoria) y/o con alguna enfermedad neuromuscular, parálisis diafragmática conocida o uso de bloqueadores neuromusculares.

En los pacientes que cumplieron con los criterios de inclusión establecidos se inició el proceso de destete ventilatorio en sus modalidades de presión soporte y prueba de respiración espontánea; además se les realizó ultrasonido diafragmático por médico residente de terapia intensiva con sistema de ultrasonido portátil Sonoscape S2 Color Doppler, utilizando un transductor convexo de $3.5 \mathrm{MHz}$ así como un transductor lineal de 9-11 MHz con el paciente en posición semisentado. Para la evaluación del grosor diafragmático se colocó el transductor perpendicularmente a la pared torácica en el octavo o noveno espacio intercostal, entre la línea axilar anterior y media. El diafragma apareció como una estructura de tres capas (dos líneas ecogénicas paralelas que representan la pleura y el peritoneo con el espacio hipoecoico central que representa el músculo diafragmático). El grosor del diafragma se midió desde el centro de la línea pleural hasta el centro de la línea peritoneal. El espesor se midió durante la inspiración final y la espiración final y posteriormente se realizó el cálculo de la variación del grosor diafragmático (VGD) mediante la siguiente fórmula: (VGD = espesor al final de la inspiración-espesor al final de la espiración/espesor al final de la espiración).

Para la evaluación de la excursión diafragmática y velocidad de contracción diafragmática se utilizó un transductor convexo colocado subcostalmente paralelo

Tabla 1: Score sonográfico predictor en el destete ventilatorio.

\begin{tabular}{lccc}
\hline Variable & 0 puntos & 1 punto & 2 puntos \\
\hline Excursión diafragmática & $\leq 10 \mathrm{~mm}$ & $11-19 \mathrm{~mm}$ & $\geq 20 \mathrm{~mm}$ \\
Velocidad de contracción & $\leq 0.8 \mathrm{~cm} / \mathrm{seg}$ & $0.9-1.3 \mathrm{~cm} / \mathrm{seg}$ & $1.4-1.7 \mathrm{~cm} / \mathrm{seg}$ \\
diafragmática & & & \\
Grosor diafragmático & $\leq 1.9 \mathrm{~mm}$ & $2-3 \mathrm{~mm}$ & $\geq 3 \mathrm{~mm}$ \\
Variación del grosor diafrag- & $\leq 20 \%$ & $21-29 \%$ & $\geq 30 \%$ \\
mático & & &
\end{tabular}

Riesgo de fracaso: 0-4 puntos. Predictor de éxito: 5-8 puntos al espacio intercostal para medir el rango del movimiento diafragmático, usando el modo $\mathrm{M}$. La velocidad de contracción se obtuvo bajo la siguiente fórmula: (VC = excursión diafragmática/tiempo inspiratorio).

Una vez realizadas las mediciones se aplicó el score realizado en la primera etapa del estudio y los resultados sonográficos no les fueron informados a los médicos responsables del tratamiento de los pacientes; el equipo de investigación sólo fue un observador descriptivo sin tomar ninguna decisión con respecto al retiro de la VM de los pacientes incluidos.

Un destete ventilatorio exitoso se definió como el paciente capaz de mantenerse sin VM por más de 48 horas después de su retiro.

El fracaso del destete de la VM se definió como el paciente que requirió nuevamente del apoyo ventilatorio dentro de las primeras 48 horas.

\section{RESULTADOS}

Se aplicó el score predictivo sonográfico para destete ventilatorio (Tabla 1) a un total de 47 pacientes con edades comprendidas entre 19 y 65 años con media para el grupo A de 37 y grupo B de 40 (Tabla 2). Se dividieron los resultados en dos grupos: el grupo $A$ comprendido por un total de 34 pacientes quienes tuvieron éxito en el proceso de destete $(72 \%)$ y el grupo $B$ formado por 13 pacientes que fracasaron en el proceso de destete ventilatorio (28\%). En cuanto al puntaje del score predictivo sonográfico para destete ventilatorio 31 pacientes (88\%) obtuvieron $\geq 5$ puntos en el score predictivo formando

Tabla 2: Datos demográficos

\begin{tabular}{lcc}
\hline Variable & Grupo A & Grupo B \\
\hline Edad & 37 & 40 \\
Sexo & & \\
$\quad$ a) Femenino & 18 & 5 \\
$\quad$ b) Masculino & 18 & 6 \\
Diagnóstico: & & \\
$\quad$ a) Quirúrgicos & 23 & 5 \\
$\quad$ b) Médicos & 10 & 2 \\
$\quad$ c) Traumáticos & 2 & 5 \\
Relación $\mathrm{PaO}_{2} / \mathrm{FiO}_{2}(\mathrm{mmHg})$ & 240 & 232 \\
Índice de $\mathrm{CROP}^{\text {Índice de Yang Tobín }}$ & 11 & 13 \\
$\quad$ Volumen Tidal $\mathrm{mL} / \mathrm{kg}$ & 29 & 32 \\
Volumen corriente $\mathrm{mL} / \mathrm{kg}$ & 7 & 7.2 \\
\hline
\end{tabular}

Tabla 3: Características grupales relacionadas con score predictivo.

\begin{tabular}{ccc}
\hline Score predictivo & Grupo A (destete exitoso) & Grupo B (fracaso) \\
\hline de 5 a 8 puntos & 31 & 4 \\
de 0 a 4 puntos & 3 & 9 \\
Total & 34 & 13 \\
\hline
\end{tabular}


Tabla 4: Características ultrasonográficas según grupos

\begin{tabular}{|c|c|c|c|c|c|c|c|}
\hline & \multicolumn{3}{|c|}{ Grupo A } & \multicolumn{3}{|c|}{ Grupo B } & \multirow[b]{2}{*}{ Valor $p$} \\
\hline & Mín. & Máx. & Media & Mín. & Máx. & Media & \\
\hline Días de intubación & 2.0 & 8.0 & 5.0 & 3.0 & 8.0 & 5.5 & 0.408 \\
\hline Excursión diafragmática (cm) & 0.8 & 5.37 & 3.0 & 0.7 & 3.2 & 1.95 & $<0.012$ \\
\hline Velocidad de contracción (cm/seg) & 0.9 & 4.4 & 2.6 & 0.3 & 3.7 & 2.02 & $<0.016$ \\
\hline Grosor diafragmático (mm) & 1.8 & 3.2 & 2.5 & 0.4 & 2.1 & 1.25 & $<0.001$ \\
\hline Porcentaje de grosor diafragmático (\%) & 18.0 & 39.0 & 28.5 & 16.0 & 32.0 & 24.00 & $<0.019$ \\
\hline
\end{tabular}

el grupo de verdaderos positivos al obtener destete exitoso; cuatro pacientes (12\%) obtuvieron $\geq 5$ puntos en el SPSDV y fracaso en el destete ventilatorio, requiriendo nuevamente soporte ventilatorio en las primeras 48 horas, conformando el grupo de los falsos positivos. De los 12 pacientes que obtuvieron un puntaje $\leq 4$ puntos, nueve pacientes $(75 \%)$ fracasaron en el destete, conformando el grupo de verdaderos negativos y tres pacientes $(25 \%)$ mostraron destete exitoso a pesar de haber obtenido puntaje $\leq 4$ en el score predictivo, conformando el grupo de los falsos negativos (Tabla 3).

Se analizaron los parámetros sonográficos en ambos grupos y los días de intubación mediante la prueba $t$ de Student para variables correlacionadas, con paquete estadístico XLSTAT 2017. Mediante este análisis se observó que no hubo diferencia estadísticamente significativa en los días de ventilación mecánica entre ambos grupos (cinco días en el grupo A y 5.5 días en el grupo B). Las mediciones sonográficas representadas en el SPSDV muestran diferencia estadística entre los grupos con destete de VM exitoso (grupo A) y el grupo con fracaso en el destete de VM (grupo B) con una media de valor en excursión diafragmática de $3 \mathrm{~mm}$ en el grupo A y $1.95 \mathrm{~mm}$ en el grupo B $(p<0.012)$. En el parámetro de velocidad de contracción diafragmática se observa una media de $2.6 \mathrm{~cm} / \mathrm{seg}$ en el grupo A y 2.02 $\mathrm{cm} / \mathrm{seg}$ en el grupo $B(p<0.016)$. En cuanto a grosor diafragmático se obtuvo una media de $2.5 \mathrm{~mm}$ en el grupo A y 1.25 en el grupo B ( $p<0.001)$. En el porcentaje de grosor diafragmático la media del grupo $A$ fue de $28.5 \%$ y del grupo B de $24 \%(p<0.019)$ (Tabla 4$)$.

La sensibilidad y especificidad del score predictivo sonográfico para destete ventilatorio se analizaron mediante estudio de pruebas diagnósticas a través del paquete estadístico Epidat 3.1, reportándose una sensibilidad de $91 \%$ y especificidad de $66 \%$ con valor predictivo positivo y negativo de 89 y $75 \%$ respectivamente, con área bajo la curva de 0.83 (Figura 1), lo que refleja que el SPSDV puede ser una herramienta predictiva valiosa para la toma de decisión clínica en el contexto de destete ventilatorio.

\section{DISCUSIÓN}

En este estudio la prevalencia de éxito y de fracaso en el destete ventilatorio fue similar a la reportada en la

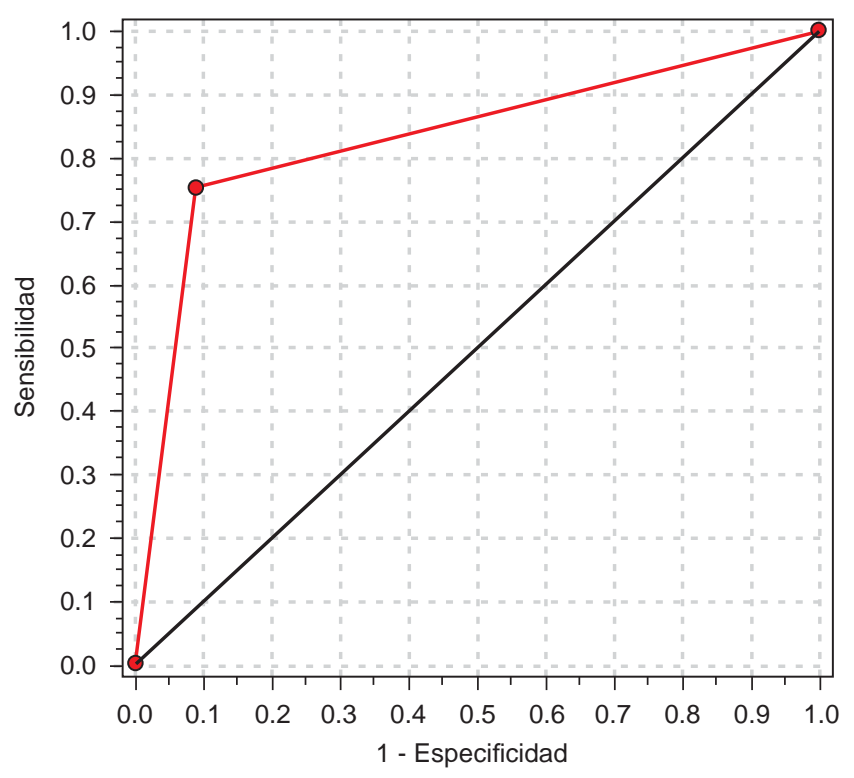

Figura 1: Área bajo la curva de 0.83. Sensibilidad de 91\% y especificidad de $66 \%$.

literatura nacional e internacional $(28 \%)$ así como el tiempo que se invierte en el proceso de destete ventilatorio. Razón por la que dicho proceso se convierte en una prioridad del médico intensivista y por lo tanto, las herramientas clínicas, ventilatorias y sonográficas son pieza fundamental para orientar hacia el éxito el destete ventilatorio. El score sonográfico predictivo de destete ventilatorio representa una herramienta útil para determinar las posibilidades de éxito en el destete de la ventilación mecánica programada, tal como se mostró con los resultados en el presente estudio. Sin embargo, también permitirá realizar un monitoreo continuo, no invasivo y sistemático en todos los pacientes bajo ventilación mecánica. De tal forma, podremos detectar de manera oportuna la alteración de cada una de las variables fisiológicas que componen el SPSDV e instalar medidas preventivas y terapéuticas en dichas alteraciones para modificar los factores adversos que condicionen fracaso en el destete ventilatorio.

El score sonográfico predictivo de destete ventilatorio puede llegar a formar parte de los bundles para el cuidado y monitoreo integral no invasivo del paciente en estado crítico. Además, es un proceso simple y pue- 
de realizarse en cualquier momento en la cabecera del enfermo con tecnología que se encuentra al alcance de todas las unidades hospitalarias y no implica mayor costo para las instituciones de salud.

\section{CONCLUSIONES}

Los resultados previamente comentados tienen relevancia en pacientes bajo ventilación mecánica, quienes son sometidos a una prueba de destete ventilatorio con el objetivo de detectar con alta sensibilidad a los que, pese a otras variables clínicas o respiratorias, tengan posibilidades de éxito en el retiro de la VM, evitando las complicaciones asociadas ya conocidas.

Con base en estos hallazgos, podemos concluir que el score sonográfico predictivo de destete ventilatorio puede formar parte del monitoreo no invasivo integral a la cabecera del paciente, pues identifica las características diafragmáticas (nos ofrece información funcional acerca del músculo) en pacientes críticos bajo VM con la ventaja de que puede repetirse como seguimiento cuantas veces sea necesario.

\section{BIBLIOGRAFÍA}

1. Osman AM. Diaphragmatic and lung ultrasound application as new predictive indices for the weaning process in ICU patients. The Egyptian Journal of Radiology and Nuclear Medicine. 2017;48:61-66.

2. Ramzy AE. Diaphragm ultrasound as a new functional and morphological index of outcome, prognosis and discontinuation from mechanical ventilation in critically ill patients and evaluating the possible protective indices against VIDD. Egyptian Journal of Chest Diseases and Tuberculosis. 2017;66:339-351.

3. Peñuelas O, Frutos-Vivar F, Fernández C, Anzueto A, Epstein SK, Apezteguía C, et al. Characteristics and outcomes of ventilated patients according to time to liberation from mechanical ventilation. Am J Respir Crit Care Med. 2011;184(4):430-437.

4. Kim WY, Suh HJ, Hong SB, Koh Y, Lim CM. Diaphragm dysfunction assessed by ultrasonography: influence on weaning from mechanical ventilation. Crit Care Med. 2011;39(12):26272630.

5. Jubran A. Critical illness and mechanical ventilation: effects on the diaphragm. Respir Care. 2006;51:1054-1061.

6. Petrof BJ, Jaber S, Matecki S. Ventilator-induced diaphragmatic dysfunction. Curr Opin Crit Care. 2016;16:19-25.

7. Hermans $\mathrm{G}$, van den Berghe $\mathrm{G}$. Clinical review: intensive care unit acquired weakness. Crit Care. 2015;19:274.

8. Matamis D, Soilemezi E, Tsagourias M, Akoumianaki E, Dimassi $\mathrm{S}$, Boroli $\mathrm{F}$, et al. Sonographic evaluation of the diaphragm in critically ill patients. Technique and clinical applications. Intensive Care Med. 2013;39(5):801-810.

9. DiNino E. Diaphragm ultrasound as a predictor of successful extubation from mechanical ventilation. Thorax. 2014;69:423427.

10. Heunks LM, Doorduin J, van der Hoeven JG. Monitoring and preventing diaphragm injury. Curr Opin Crit Care. 2015;21:34-41.

Patrocinio: No se utilizó ningún recurso fuera del Instituto Mexicano del Seguro Social.

Conflictos de interés: El autor declara no tener ningún conflicto de interés.

Correspondencia:

Dra. Alma Erika Hernández Plata. Apolo XI 101-A Int 4. Colonia Futurama Monterrey. León, Guanajuato. 37180.

E-mail: kika_1980@hotmail.com 